Radial and Nonradial Pulsations as Probes of Stellar Physics

ASP Conference Series, Vol. 259, 2002

C. Aerts, T.R. Bedding, \& J. Christensen-Dalsgaard, eds.

\title{
Frequency Analysis of a Delta Scuti Type Component in the Eclipsing Binary Y Cam
}

\author{
S.-L. Kim ${ }^{1}$, J.-W. Lee ${ }^{2}$, J.-H. Youn ${ }^{1}$, S.-G. Kwon ${ }^{1}$, Chulhee Kim ${ }^{3}$
}

Abstract. We present CCD photometric results of the eclipsing binary $Y$ Cam with a pulsating component. The dominant pulsation frequency of $15.0473 \mathrm{c} \mathrm{d}^{-1}$ has not changed over 39 years, but its amplitude decreased to about half. The pulsational properties of the second frequency showed great differences between Broglia \& Conconi (1984)'s result and ours.

Delta Scuti type pulsating components in eclipsing binary systems are attractive observing targets from an asteroseismological point of view because they allow us precise determination of masses and radii for each component, detection of sectoral modes and spatial filtration, etc. (Mkrtichian et al., these proceedings). Nevertheless, few stars have been reported so far and only two stars, AB Cas (Rodríguez et al., 1998) and RZ Cas (Ohshima et al., 2001), were studied in detail.

Table 1. Results of multi-frequency analysis

\begin{tabular}{lcccccc}
\hline \hline Reference & H.J.D. & $\mathrm{f}_{1}\left(\mathrm{c} \mathrm{d}^{-1}\right)$ & $\mathrm{A}_{1}(\mathrm{~V})$ & $\mathrm{f}_{2}\left(\mathrm{c} \mathrm{d}^{-1}\right)$ & $\mathrm{A}_{2}(\mathrm{~V})$ & Residual \\
\hline This study & $51855-2061$ & 15.0473 & $0^{\mathrm{m}} 0052$ & 18.2852 & $0^{\mathrm{m}} 0039$ & $0^{\mathrm{m}} 0069$ \\
BC84 Group I & $37582-7760$ & 15.0473 & $0^{\mathrm{m}} 0122$ & 14.5890 & $0^{\mathrm{m}} 0060$ & $0^{\mathrm{m}} 0068$ \\
BC84 Group II & $44901-5028$ & 15.0473 & $0^{\mathrm{m}} 0119$ & 14.6593 & $0^{\mathrm{m}} 0103$ & $0^{\mathrm{m}} 0079$ \\
\hline
\end{tabular}

We carried out CCD photometric observations for 17 nights from November 2000 to May 2001, using a PM512 CCD camera attached to the $61 \mathrm{~cm}$ telescope at the Sobaeksan Optical Astronomy Observatory in Korea. Instrumental magnitudes were obtained using the ADPS (Automatized Differential Photometry System; Park, 1993). A total of 1065 V-band differential magnitudes were collected. Phase diagram and residuals after fitting an eclipsing light curve solution are shown in Fig. 1.

We applied the multiple frequency analysis to investigate the small amplitude oscillation features, and confirmed their presence out of eclipse and in the secondary minimum (orbital phase from 0.1 to 0.9 ; Fig. 2). Detailed results of

\footnotetext{
${ }^{1}$ Korea Astronomy Observatory, Daejon, 305-348, Korea

${ }^{2}$ Dep. of Astronomy and Space Science, Chungbuk Nat. Univ., Cheongju, 361-763, Korea

${ }^{3}$ Dep. of Earth Science Education, Chonbuk Nat. Univ., Chonju, 561-756, Korea
} 


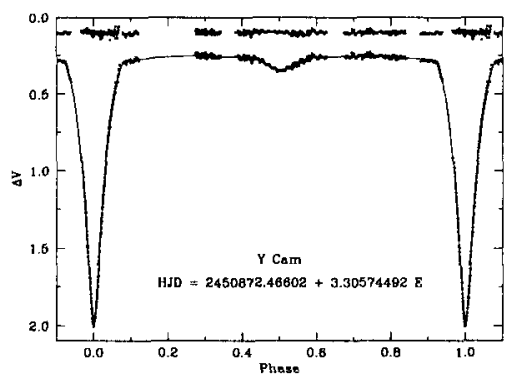

Figure 1. Phase diagram (lower) and residuals (upper).

the analysis are listed in Table 1 , including the previous results by Broglia \& Conconi (1984) for comparison. The first frequency of $15.0473 \mathrm{c} \mathrm{d}^{-1}$ turned out to be unchanged over about 39 years, but its amplitude decreased to about half. The second frequency and its amplitude showed great differences. In conclusion, we suggest that the pulsating component in the eclipsing binary Y Cam has undergone amplitude variations and/or mode changes.

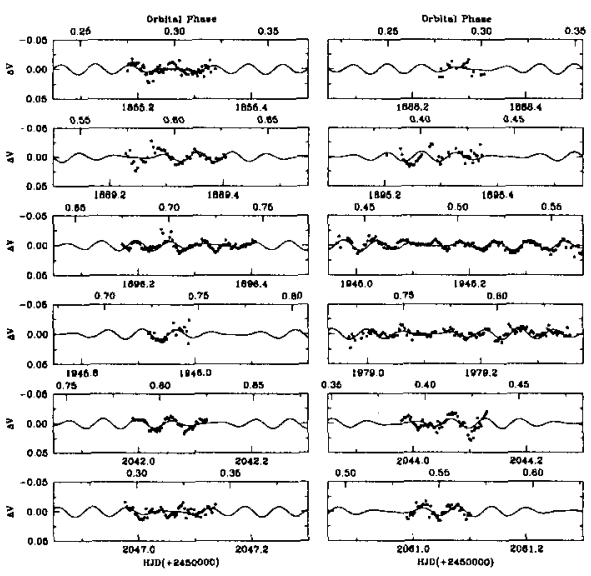

Figure 2. Light variations showing small amplitude pulsation.

\section{References}

Broglia, P. \& Conconi, P. 1984, A\&A, 138, 443

Ohshima, O., Narusawa, S.-Y., Akazawa, H., Arai, K., Fujii, M., et al. 2001, AJ, 122, 418

Park, N.-K. 1993, Publications of the Korean Astronomical Society, 8, 185

Rodríguez, E., Claret, A., Sedano, J. L., García, J.M., \& Garrido, R. 1998, $\mathrm{A} \& \mathrm{~A}, 340,196$ 